\title{
METAL ABUNDANCES OF MAGELLANIC CLOUD CLUSTERS
}

\author{
D. GEISLER, A. MCWILLIAM, N. SUNTZEFF \\ Cerro Tololo Inter-American Observatory \\ Casilla 603 \\ La Serena \\ Chile \\ M. MATEO, J. GRAHAM \\ Observatories of the Carnegie Inst. of Washington \\ L. PASQUINI \\ European Southern Observatory
}

\begin{abstract}
We have obtained Washington CCD photometry with the CTIO $4 \mathrm{~m}$ and $1.5 \mathrm{~m}$ for $\sim 50$ intermediate-to-old age star clusters in the Clouds. The data extend to near or below the main sequence and provide excellent photometry for the giants, from which precise (internal errors $<0.1$ dex) mean cluster abundances can be determined. We present data for several of the clusters and discuss the results. Intermediate resolution spectra have also been obtained for some 16 clusters with the CTIO 4m ARGUS multiple-object fibre-fed spectrograph. Finally, we have also obtained high dispersion $(R \sim 20,000)$ échelle spectra for several of the brighter giants in a small sample of Large Magellanic Cloud (LMC) clusters. Detailed elemental abundances derived from these spectra will be presented. These data will help refine our knowledge of the age-metallicity relation in the Clouds.
\end{abstract}

\section{Introduction}

Magellanic Cloud clusters offer a unique opportunity to study chemical abundances in two galaxies whose chemical evolution and cluster formation histories are clearly different from that in our own Galaxy. Unfortunately, good abundance determinations are still lacking for many Magellanic Cloud clusters despite their crucial role in age-metallicity and distance calibrations. An important program to address this issue has been undertaken by Schommer et al. (1990). We have initiated a large-scale program to determine abundances for a large sample of clusters using a variety of photometric and spectroscopic techniques. The data have all been obtained and are in various stages of reduction. Some preliminary results will be presented here.

\section{Washington Photometry}

Washington CCD photometry has been shown to be a useful technique for investigating metalabundances of a large number of giants in MC clusters (Geisler 1987). We (D. Geisler and M. Mateo) have now obtained Washington CCD observations for a total of 16 SMC and 34 LMC clusters, using the CTIO $4 \mathrm{~m}$ and $1.5 \mathrm{~m}$ telescopes. The data are being reduced with the automatic psf-fitting routine DoPHOT (Mateo and Schechter 1990). The photometric errors for the giants are typically $<0.02$ mag and allow very precise mean abundances to be determined from $\sim 30-60$ giants 
per cluster. The intrinsic abundance spreads in the clusters are generally $<0.1$ dex. However, the determination of the mean absolute cluster abundances are more uncertain. Both aperture correction and photometric zeropoint uncertainties, as well as sensitivity to reddening, inhibit the determination of accurate abundances. For clusters with $[\mathrm{FE} / \mathrm{H}] \leq-1$ (most of the sample), the total uncertainty in the abundance due to all of these sources is $\sim 0.3$ dex. For the older, more metalpoor clusters, the abundance errors become larger.

\section{Medium resolution spectroscopy}

The CTIO 4m ARGUS multi-object fibre-fed spectrograph is ideally suited to studying the compositon of many MC cluster giants simultaneously. We (D. Geisler, N. Suntzeff, J. Graham and M. Mateo) have enjoyed two successful runs, obtaining data for $16 \mathrm{LMC}$ clusters. Despite the 24 fibres available, only $\sim 10$ spectra can be obtained per cluster because of their small angular size. The spectra range from $\sim 4900-6400 \AA$, with a resolution of $\sim 5 \AA$, and will provide us with $\mathrm{Mg}$, $\mathrm{Na}, \mathrm{Fe}$ and $\mathrm{TiO}$ indices from which to derive abundances. Unfortunately, we are not able to present any spectra here as all the reductions were recently inadvertently destroyed before being backed up! But the data (still alive!) look good...

\section{High resolution spectroscopy}

Finally, we (D. Geisler, A. McWilliam and L. Pasquini) have obtained high-resolution (R 20,000) échelle spectra of a few giants in each of several clusters with the CTIO $4 \mathrm{~m}$ and ESO $3.6 \mathrm{~m}$. The spectra are centred near $7000 \AA$. We have observed a small sample of young, intermediate-age and old clusters (including two stars in NGC 2257!). A full model atmosphere curve-of growth analysis will be applied to derive detailed elemental abundances.

\section{References}

Geisler, D. (1987), A. J. 93, 1081.

Mateo, M., Schechter, P. (1990), Proceedings of the First ESO Workshop on Data Analysis, in press.

Schommer, R.A., Olszewski, E., Suntzeff, N. B. (1990), this volume. 